\title{
A Network Model for Deep Bed Filtration of Solid Particles and Emulsion Drops
}

\begin{abstract}
A network model has been developed to simulate the flow of emulsions and solid particles through porous media. Particle deposition due to direct interception, as well pore plugging by straining are accounted for in the model. The effects of two important factors - the ratio of particle size to pore size, and the fluid velocity - on particle deposition are also investigated. The strength of the model lies in its ability to predict accurately effluent concentration profiles, permeability changes occurring during deep bed filtration, and the evolution of the filter coefficient with time. Model predictions for different particle and pore size distributions of both solid and emulsion particles are in agreement with experimental data.
\end{abstract}

\author{
S. D. Rege, H. S. Fogler \\ Department of Chemical Engineering \\ University of Michigan \\ Ann Arbor, Ml 48109
}

\section{Introduction}

Deep bed filtration (DBF) is a process in which particles present in the flowing suspension deposit at different locations within the porous medium. DBF has been extensively studied for several years since it is of significance in many industrial applications. For example, DBF is of ten used to remove colloidal particles from dilute liquid suspensions. A major application of DBF is waterflooding operations for enhanced oil recovery. Water that is injected into the formation (or oil-bearing porous medium) during these operations (to increase the oil production), generally contains particles of various sizes. Deep bed filtration of these particles can significantly reduce the permeability of the porous medium. In these applications it is important to predict the effluent particle concentrations, and the resulting decline in the formation permeability.

It is usually necessary to prefilter the water before injection, to prevent significant damage to porous medium because of DBF. The extent of prefiltration depends on two factors: the cost of prefiltration, and the amount of formation damage that is permissible. While it is cheaper to prefilter only the relatively large particles (in comparison to filtering both large and fine particles), the resulting damage will consequently be relatively higher. On the other hand, to filter every particle would be quite expensive, and in many cases impractical. Consequently a balance must be achieved between the cost of prefiltration and formation damage.

DBF is generally characterized by three parameters:

1. The filter coefficient, which governs the rate of deposition

2. The filtration efficiency, which determines the extent of removal of particles from the suspension
3. The flow restriction parameter, which relates the amount of deposition to the permeability decline

Reviews by Tien and Payatakes (1979) and Herzig et al. (1970) give an excellent discussion of some of the previous deep bed filtration models. These and other models can be classified as follows:

- Empirical

- Trajectory analysis

- Stochastic

- Network

While a detailed description of these models is not possible here, we discuss them briefly with a view to understanding their limitations.

\section{Empirical models}

The empirical models (Heertjes and Lerk, 1967; Ives, 1960; Ison and Ives, 1969) consider the porous medium to be a closed system within which deposition occurs. A mass balance equation for the particles takes the form,

$$
\frac{\partial \sigma}{\partial t}+u \frac{\partial c}{\partial x}=0
$$

In order to solve this equation a rate law for deposition is required. Several forms of this rate law have been suggested in the literature (see reviews by Tien and Payatakes, 1979; Herzig et al., 1970) and an example (Iwasaki, 1937) is shown below:

$$
\frac{\partial \sigma}{\partial t}=u \lambda c
$$

where $\lambda=\lambda_{0} f(\boldsymbol{P}, \sigma) ; \boldsymbol{P}$ is an unknown parametric vector. 
More exact forms for Eq. 1 and 2 have been discussed by Horner et al. (1984). Equations 1 and 2 can be solved to yield the effluent concentration and the amount of deposition as a function of distance and time. The value of $\lambda_{0}$ is obtained by fitting the predicted effluent concentration history to the experimental data at initial times. The parametric vector $\boldsymbol{P}$ depends on factors such as grain density, fluid velocity, and so on. Attempts to quantify this vector a priori, have been unsuccessful.

The pressure drop changes are then related to the amount of deposition by empirical correlations, such as (lves and Pienvichitr, 1965),

$$
\frac{(\partial P / \partial x)}{(\partial P / \partial x)_{o}}=\frac{1}{\left(1-\beta_{2} * \sigma\right)^{\beta_{1}}}
$$

where $\beta_{1}, \beta_{2}$ are parameters.

While the empirical approach is simple, it does not give significant insight into the physics of the deposition process. For example, it fails to take into account factors such as the pore size and particle size distributions or the deposition morphologies. These models cannot predict the permeability response or effluent concentration profiles during deep bed filtration. Several parameters, such as the filter coefficient and the constants, $\beta_{1}, \beta_{2}$, which relate the permeability change to the amount of deposit, are determined by fitting the experimental data.

The empirical models developed by Gruesbeck and Collins (1982) and Maroudas and Eisenklam (1965a,b) consider two different types of pores in the medium: those in which blocking occurs and those in which surface deposition occurs due to direct interception. These models require additional information, in some cases as many as six adjustable parameters (such as the blocking coefficient, the fraction of plugging and nonpulgging pores, and so on), which can be determined by fitting the experimental data. The values of these parameters change with varying pore size and particle size distributions, and consequently these models too are not predictive in nature.

A recent model, which combines the empirical model approach with the model of Gruesbeck and Collins (1982), has been developed by Soo and Radke (1986). Here the filtration of dilute stable emulsions is modeled, building on earlier work on solid particles. Certain relationships and parameter values obtained from the unit-bed model and trajectory analysis of Payatakes et al. $(1973,1974)$ have also been used in this model.

Soo and coworkers (Soo and Radke, 1986; Soo et al., 1986) characterize the filtration process by three empirical parameters that have well-defined meanings. These parameters are:

An average filter coefficient of a clean bed, $\lambda$

An average flow redistribution parameter, $\alpha$

An average flow restriction parameter, $\beta$

The system is mathematically represented in terms of these parameters as

$$
\begin{gathered}
\frac{\partial \sigma}{\partial t}=\lambda\left(1-\alpha \sigma / \phi_{0}\right) u c \\
\frac{K_{x}}{K_{0}}=1-\beta \sigma / \phi_{0}
\end{gathered}
$$

where $K_{x}$ is the local permeability at time $t$, and $K_{0}$ is the initial permeability. This equation is solved using the appropriate initial and boundary conditions, to yield the retention and effluent concentration profiles. The values of the three parameters, $\lambda, \alpha$, and $\beta$, are then determined by fitting the experimental data for effuent concentration profiles and permeability response. The values of $\lambda, \alpha$, and $\beta$, were found to vary from run to run; their trends are discussed by Soo et al. (1986). The authors also try to estimate these parameters a priori. The estimation, however, involves the use of some empirical relationships and three other parameters:

$\lambda_{i}$, the filter coefficient in the interception pores (pores in which straining does not occur, i.e., nonplugging type)

$\zeta$, the fraction of monolayer surface coverage

$D_{v}$, the transition diameter between the straining and interception pores

(In these parameters the symbol $\theta$ was used rather than $\zeta$ in the original paper. Here, we use $\zeta$ to avoid confusion with the symbol $\theta$, which is used in our work in the relationship for the capture probability.)

$\lambda_{i}$ is obtained from the unit bed element results of Payatakes et al. (1974). This value ranges from $10^{-3} / L$ to $10^{-2} / L$ depending on the particle to pore size ratio ( $L$ is the thickness of a unit bed element). Even over this range, the estimated values of $\lambda$ show deviation from the experimental values, especially for larger drop sizes (see Figure 4 of Soo et al., 1986). $D_{t}$ and $\zeta$ are obtained by fitting empirical relationships for $\alpha$ and $\beta$ to experimental values (which in effect were also obtained by fitting model equations to the concentration profiles and the permeability response). As noted by the authors, several of the parameters are not unique although the range over which they can vary is small.

\section{Trajectory analysis model}

The trajectory analysis model (Payatakes et al., 1973) represents the porous medium as unit bed collectors. These collectors can be of different geometric shapes, such as spherical, constricted tubes, and others. The trajectory of each particle within the unit bed is calculated using streamline functions, combined with several forces (such as gravitational, inertial, hydrodynamic, electric double layer, and van der Waals forces) which act on the particle. This analysis is sophisticated and has made a significant advancement in understanding the deep bed filtration process at the pore level.

While this approach has been fairly successful in predicting filter coefficients and filtration efficiencies, it has some limitations. First, it does not accurately predict the permeability changes due to DBF. A possible cause for this is that the correlation given for permeability change depends on the Kozeny-Carman equation, which is not applicable in this case; the KozenyCarman equation assumes a capillary bundle-of-tubes model and ignores the interconnectivity between pores. Second, in the simulation (Tien et al., 1979) it is assumed that the deposition morphologies undergo a sudden transition from smooth deposition to blocking type deposition when the amount of deposit reaches a value of $\sigma_{\text {tran }}$. The values of $\sigma_{\text {tran }}$ and other parameters (critical interstitial velocity, and porosity of deposit) required to calculate the pressure drop changes, are approximated based on previous experimental data of other researchers (Deb, 1969; Maroudas and Eisenklam, 1965a,b). The direct us of these values for different deposition conditions is questionable, as is their accuracy. Third, this approach primarily considers monodispersed particles, while suspensions injected into the porous media generally contain polydispersed particles. (Pendse and 
Tien (1982) consider polydisperse particles in their recent work on aerosol filtration. Here, particle capture occurs primarily due to inertial forces.) Recent work by Mackie et al. (1987) does not involve any sudden transition and does take into account the polydispersity of the particles.

\section{Stochastic models}

The stochastic models (Litwiniszyn, 1966; Hsu and Fan, 1984; Fan et al., 1985) use a probabilistic approach in considering deep bed filtration. The relatively recent model developed by Fan et al. (1985) simulates the performance of a deep bed filter by a birth-death process that takes into account the blockage of pores as well as scouring (removal) of deposited particles. A birth event represents an open pore getting blocked, while a death event represents a blocked pore getting unblocked due to scouring of particles. The mean number of blocked pores at any given moment is determined by solving probability equations, and is then related to the fluid velocity and the permeability ratio. This model has two parameters, which are obtained by fitting the experimental data. While the stochastic models are mathematically simple, they too do not consider the effects of pore size and particle size distributions, or the deposition morphologies, on the filtration process. In fact, the analysis is developed for pores of uniform sizes. In addition, the model does not attempt to predict the effluent concentration profiles.

\section{Network models}

The network model was first used to simulate formation damage by Todd et al. (1984), who used an unbiased random walk to move the particles through the network. Two methods of capture were employed in the simulations: random capture and capture by $1 / 3$ rd $-1 / 7$ th geometric rule. Model predictions using both these techniques did not match the experimental data, which was attributed by the authors to the inadequacies of the capture mechanisms. Furthermore, an unbaised random walk model does not account for the fluid flow effects on particle movement (termed flow biasing), which has been shown (Rege and Fogler, 1987) to be important especially for straining dominated particle capture.

Another model, which uses the effective-medium approximation (EMA) of the network models (Kirkpatrick, 1973; Koplik, 1981, 1982), has been developed by Sharma and Yortsos $(1987 \mathrm{a}, \mathrm{b}, \mathrm{c})$. This model combines the population balance equations with an in-depth study of the rates of particle release and deposition. Two types of particle capture mechanisms have been considered: straining and smooth deposition. The permeability changes resulting from particle capture are then calculated using EMA. The model attempts, in general, to describe the deposition kinetics, and also to predict the effluent concentration profiles, the permeability response, and the filter coefficient.

While this is a good model for particle capture, it has certain limitations that need discussion. First, in determining permeability changes it does not consider different deposition morphologies for relatively small particles; that is, it does not distinguish between smooth deposition and dendrite formation. Second, the rate of deposition, which is calculated using the Levich solution for mass transfer of Brownian particles, is not applicable for relatively large particles $(>2 \mu \mathrm{m})$. For these particles the ratio of particle size and throat size is an important factor in determining deposition rate; this is not accounted for in the analysis of Sharma and Yortsos (1987a,b). Third, use of the effective-medium theory, in which all bonds in the network are replaced by bonds of uniform conductance, provides only approximate results for the permeability change of the system. EMA also assumes that for all pore throats of a given size, the fluid velocity is the same, no matter what their location is in the network. This assumption is questionable, especially when the pore throat distribution is relatively wide. Another limitation of the model is that it assumes the fraction of particles of a particular size, captured by straining, to be the same along the entire length of the porous media. No rationale has been provided to justify this assumption.

A comparison of the Sharma and Yortsos model predictions with experimental data indicates deviations, especially at lower values of the permeability ratios (e.g., Figures 13 and 14 of Sharma and Yortsos, 1987b). Also, the values of certain dimensionless variables (Sharma and Yortsos, 1987a,b) have been used as adjustable parameters (even though in theory these can be determined a priori) to fit the permeability response curves at initial times.

In the present study we use the network model in its entirety and track the individual particles as they move through the network. This approach is relatively more accurate than the effective-medium approximation. Also, the current model can simulate straining and deep bed filtration occurring simultaneously. Another important feature of this model is that it accounts for differences in deposition morphologies observed between emulsion droplet capture and solid particle capture. Model predictions of the permeability response and effluent concentraticn profiles show good agreement with the experimental data of Soo and Radke (1984, 1985), and Baghdikian et al. (1987).

\section{Model Formulation}

In this study we use a network model representation of porous media. The network model, which was first introduced by Fatt (1956), consists of interconnected bonds which represent the pore throats in the medium. The bonds intersect at points of mixing called nodes. Network models can predict certain static and dynamic properties of porous media, such as capillary pressure characteristics and relative permeability curves, which are similar to those observed experimentally. It also has interconnecting pathways which make it structurally similar to porous media. The network model has previously been used to study several processes, such as dispersion (Sahimi, et al., 1983, 1986a, b), fluid displacement (Simon and Kelsey, 1971, 1972), carbonate acidizing (Hoefner and Fogler, 1988), particulate straining (Rege and Fogler, 1987), and others (Yortsos and Sharma, 1986; Siegel and Langer, 1986).

Several types of networks have been used in the literature, as shown in Figure 1. Due to its random nature, the Voronoi type of network most closely resembles the porous media. However, the regular triangular network, has also been shown to possess certain properties similar to the Voronoi lattice, (Jerauld et al., 1984) and to the real porous media (Fatt, 1956). Additionally, flow calculations on such a regular network are computationally more efficient, and good results (using triangular networks) have been obtained previously for straining dominated particle capture by Rege and Fogler (1987), and later by Imdakm and Sahimi (1987). The regular triangular network will therefore be used to represent the porous media in this study as well. 


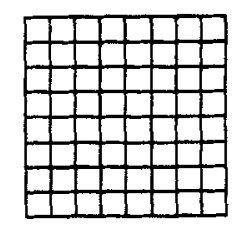

Square

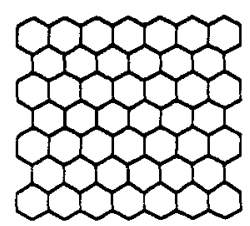

single Hexagonal

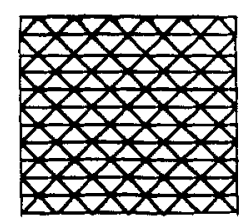

Triangular

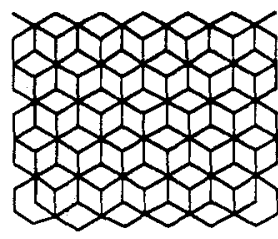

Double Hexagonal
Figure 1. Types of network models.

In order to simulate fluid flow in the network we first assign a pore throat diameter distribution to the bonds in the network. The actual form of the distribution used in the network varies depending on the porous sample in which particle capture is being studied. The pore size distribution of the sample under consideration can be obtained by mercury porosimetry or photomicrographic techniques (Dullien and Batra, 1970). This distribution is then assigned randomly to the bonds in the network and serves as an approximate yet realistic representation of the porous media. If different sets of random numbers are selected, then one obtains networks with statistical variations, called realizations. Simulations are carried out on several realizations and mean values are generally reported.

The bond lengths can be selected to be constant, randomly assigned, or related in some manner (directly or inversely proportional) to the diameter. Since a sensitivity analysis showed that the choice of these lengths was not critical in our study, we consider the bonds to have a constant length equal to the mean diameter of the pore size distribution; this is equivalent to assigning the pore size distribution randomly to the bond lengths.

The fluid flow through the individual bonds is then calculated by simultaneously solving mass balance equations for the fluid at every node. Once the flow distribution within the network is estimated, we can simulate particle entrainment and entrapment. To obtain realistic results, that is, without any edge or size effects, it is necessary to use networks of relatively large sizes. In order to handle large arrays and perform efficient computations, the simulations were carried out on the Cray X-MP supercomputer at the Pittsburgh Supercomputing Center.

Particle movement and capture is simulated by randomly generating particles from a given distribution at the entrance to the network, and then transporting these particles through bonds where they may be captured, or may pass through. Particle movement is governed by two fundamental concepts: flowbiased probability and wave front movement. As particles pass through a bond, they arrive at nodes which are points of mixing. Since several paths may carry fluid away from the node, it is necessary to choose one of these paths for the particle to flow through. This particular path is determined by flow-biased probability, which biases the movement of the particle toward the path with a greater flow. Due to the stochastic nature of the simulation, paths with lower flow rates may also get selected, although the probability of that occurring may be small. The wave front movement ensures that several particles are simultaneously transported through the network. Details regarding both the above concepts have been given elsewhere (Rege and Fogler, 1987). These concepts have also been used previously by Sahimi et al. (1983) in the context of hydrodynamic dispersion of injected tracer.

In the current model particle capture can occur due to straining as well as direct interception. Straining occurs when the particle size is larger than the bond diameter selected for it to pass through. Straining plugs up the bond, thereby diverting the flow to other paths. Direct interception, that is, deposition of a particle on a pore wall (bond), occurs as a result of several forces, such as hydrodynamic, gravitational, and the likes, acting on the particle.

To simulate deep bed filtration in the network model it is necessary to determine:

1. The probability of capture of particles smaller than the pores they travel through

2. The change in pressure drop due to particle deposition

In the current model the probability of capture for any particle passing through a pore larger than itself is calculated using a modified form of the relationship first used by Stein (1940):

Initial capture probability

$$
=4^{*}\left[\left(\theta a / R_{0}\right)^{2}-\left(\theta a / R_{0}\right)^{3}\right]+\left(\theta a / R_{0}\right)^{4}
$$

where $a=$ radius of particle

$R_{0}=$ radius of bond

$\theta=$ parameter

This relation determines the fraction of flow in an annulus of size $\theta a$, under laminar flow conditions, as shown in Figure 2. If for certain values of $\theta, a$, and $R_{0}, \theta a$ is greater than $R_{0}$, the probability of capture is set equal to 1.0 and the particle is captured.

$\theta$ is a lumped parameter that takes into account the effect on deposition of several forces mentioned previously. For example, a high value of $\theta$ suggests that the surface conditions are favorable for deposition, because particles in a relatively large annulus can be captured. Similarly, a relatively low value of $\theta$ denotes unfavorable deposition conditions. The boundary of the annulus as determined by $\theta$ is equivalent to the limiting trajectory concept in the model of Tien and Payatakes (1979).

Factors that affect the value of $\theta$ are fluid velocity, ionic strength, $\mathrm{pH}$, fluid properties, and particle density and concen-

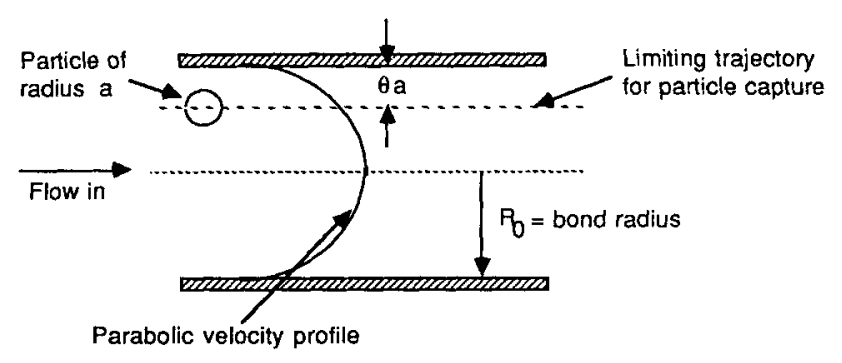

Figure 2. Particle capture probability is equivalent to fraction of total flow in annulus between $R_{0}$ and $\boldsymbol{R}_{0}-\theta a$. 
tration. Here we attempt to consider the fluid velocity effects on $\theta$ by postulating a relationship of the form,

$$
\theta=\theta_{0} \exp \left(-v / v^{*}\right)
$$

where $\theta_{0}$ is a constant dependent on ionic conditions $v$ is the fluid velocity in the bond $v^{*}$ is a critical velocity

The choice of this relationship stems from experimental observations of Gruesbeck and Collins (1982), and details are explained elsewhere (Rege, 1988). The exponential term accounts for the effects of velocity on the capture probability. The value of $v^{*}$ can be determined approximately, either from experimental runs (shown later in this paper), or based on the approaches of Spielman and Fitzpatrick (1973), Spielman and Cukor (1973), and Mackie et al. (1987). For relatively low values of $v / v^{*}, \theta=\theta_{0}$ throughout a run, since the exponential term is in the neighborhood of 1.0 and is not significantly affected by changes in the velocity. For relatively high values of $v / v^{*}$ (say, $v / v^{*}>0.15$ ), an increase in the fluid velocity $v$ causes a decrease in the value of $\theta$. This is in qualitative agreement with experimental data, where high velocities cause relatively low deposition because of increased shear forces.

While $\theta_{0}$ is a lumped parameter, its value is expected to be constant with changes in the particle size or pore size distributions, and inlet particle concentration (for dilute suspensions). The value of $\theta_{0}$ is determined by performing a single experimental run (at low velocities, such that $v \ll v^{*}$ ), and by adjusting $\theta_{0}$ such that the model predictions match the experimental data for that run. Using this value of $\theta_{0}$, the model can then predict the effluent concentration profiles and the permeability response for subsequent runs in which the size distributions or the inlet concentrations may be varied. The value of $\theta_{0}$ is primarily affected by changes in the ionic strength and $\mathrm{pH}$.

The evolution of capture probability with time for emulsion droplets and solid particles is calculated as follows. When a particle deposits in the bonds, the fluid exerts a drag force on the particle, which increases the resistance to flow in that bond; this is equivalent to a reduction in the bond radius. The new effective bond radius (which can be indirectly calculated based on Eqs. 8 and 9 below), is then used in Eq. 7 instead of the initial radius, $R_{0}$. Since in reality the capture probability would also depend on the exact deposition morphology (and not just the effective diameter), the method used here is a first approximation.

When a particle is captured in the bond the resistance of that bond increases proportionally to the drag force experienced by the particle. The resulting pressure drop increase due to the particle can be approximately calculated by the following relationship (Happel and Brenner, 1973):

$$
\Delta P_{\text {particle }}=\frac{12 \eta a U_{0}}{R_{0}^{2}}\left[1-\left(1-a / R_{0}\right)^{2}\right]^{2} \cdot K_{1}
$$

where

$$
\begin{aligned}
& \begin{aligned}
K_{1}= & \frac{1-(2 / 3) \cdot\left(a / R_{0}\right)^{2}-0.202\left(a / R_{0}\right)^{5}}{1-2.1\left(a / R_{0}\right)+2.09\left(a / R_{0}\right)^{3}} \\
& \quad-1.71\left(a / R_{0}\right)^{5}+0.73\left(a / R_{0}\right)^{6}
\end{aligned} \\
& \begin{aligned}
U_{0}= & \text { centerline velocity } \\
\eta= & \text { viscosity }
\end{aligned}
\end{aligned}
$$

This equation accounts for wall effects on the drag force and holds for fairly large $a / R_{0}$ ratios in laminar flow through cylindrical tubes. The total pressure drop through the bond is given by

$$
\Delta P_{\text {total }}=\Delta P_{\text {clean tube }}+\Delta P_{\text {particle }}
$$

The new effective radius after $N$ particles have deposited is given by

$$
\frac{1}{R_{\text {new }}^{4}}=\frac{1}{R_{0}^{4}}+0.1875 \sum_{i=1}^{N} \frac{a_{i}}{L} \cdot\left[1-\left(1-a / R_{0}\right)^{2}\right]^{2} \cdot K_{1}
$$

The morphology of deposition varies depending on whether the particles are solids or emulsion droplets. Soo and Radke (1984) have shown that emulsion droplets, unlike solid particles, do not deposit on other droplets to form dendrites but deposit as a monolayer on the grain surfaces. For this case, the radial distance of the particle from the center of the bond is known. This distance is used to calculate the velocity, and thereby the drag force, experienced by an emulsion drop. Furthermore, once the available surface area is covered, the bed is considered to be saturated and no further deposition can occur. For the case of solid particles, deposition occurs at the wall and also on other particles present in the bond, leading to dendrite formation (Tien et al., 1979; Soo and Radke, 1985). Since it is not possible to determine the location of every deposited particle within a bond, we assume that the deposited particles experience the average fluid velocity within that bond. Since particles initially deposit on the wall, and experience a velocity less than the average, the model would predict a slightly higher decline in the permeability ratio during the initial stage of the run.

It should be noted that the drag force calculations are approximate due to two reasons:

1. As particle deposition progresses, the parabolic velocity profile in the bonds may no longer be maintained, and we do not account for this in the model.

2. Effects of particle-particle interaction are not accounted for in the pressure drop relationship, Eq. 8 .

We shall see later that neglecting these effects is of little consequence in making accurate predictions.

As particle capture occurs, the overall resistance to fluid flow in the network increases. On the bond level, particle capture results in flow redistribution, and this is inherently accounted for in the network model. Mass balance equations are continually re-solved to give the current pressure drop and thereby the permeability ratio, as a funtion of the pore volumes injected. Effluent concentration profiles are also monitored throughout the simulation. Since we track the individual particles in the model, it is also possible to determine the importance of straining in relation to deep bed filtration for a given set of experimental conditions.

An overall description of the model is presented in the algorithm shown in Figure 3.

\section{Results and Discussion}

Solid particles are injected into the network at time $t=0$. As the simulation proceeds, more particles are injected and we track these particles through the network. We also monitor the effluent concentration profiles, the permeability response, and the filter coefficient ratio. Simulations are performed on rela- 


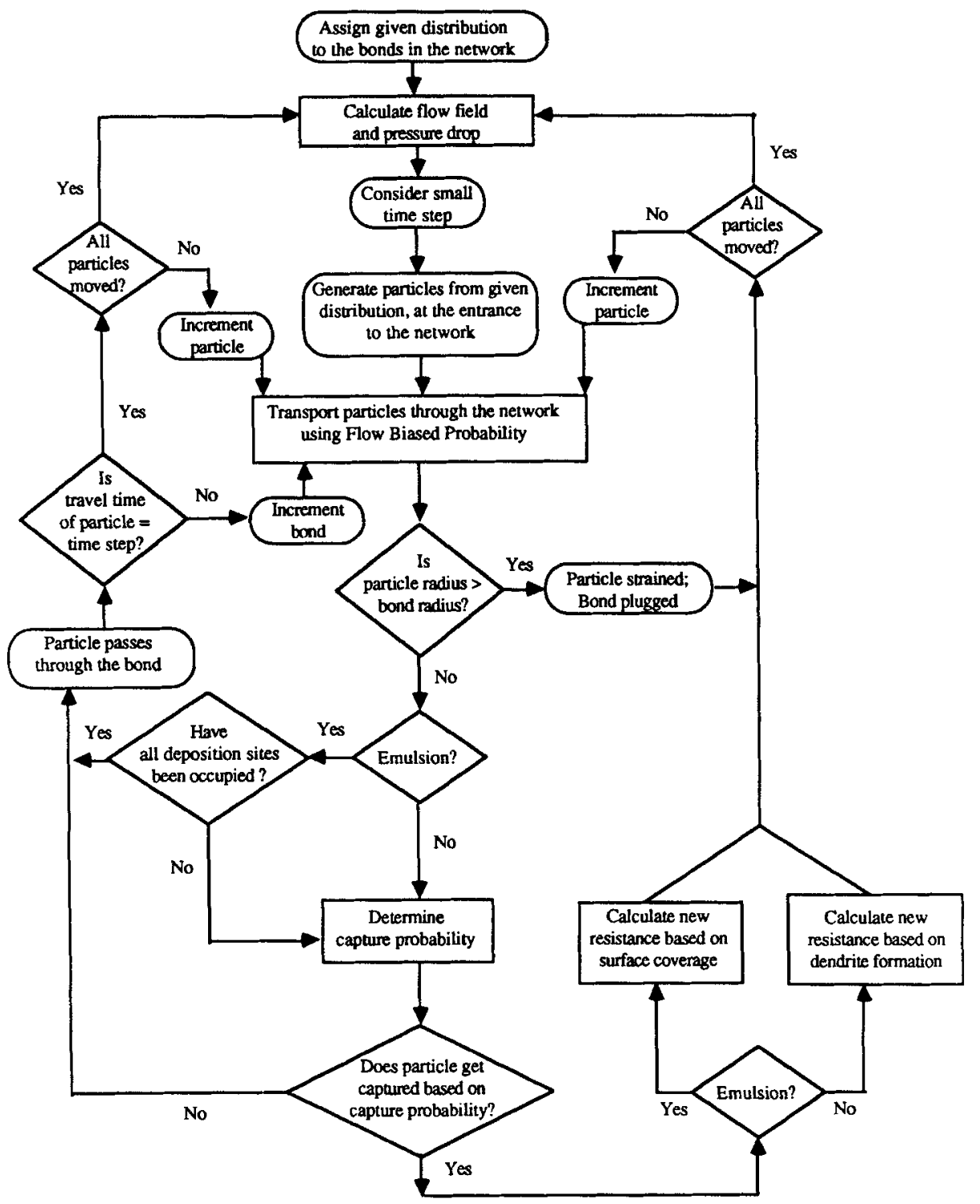

Figure 3. Flowsheet describing essential features of the model.

tively large networks $(60 \times 60)$ on the Cray-X/MP supercomputer. Periodic wraparound boundary conditions are used at the sides of the network, and simulations are performed at constant injection rate. Since the simulation is stochastic in nature, Monte Carlo runs (eight, in general) are carried out on five different realizations of the network. The results reported below are the average values obtained after running the simulations in the above manner.

\section{Permeability ratio and effuent concentration}

Using the current model we first simulate solid particle capture for monodispersed pores and particles. The mean pore and particle diameter, in the following examples, are arbitrarily selected to be 25 and $1 \mu \mathrm{m}$, respectively. The effect of $\theta$ on the permeability response is first investigated (for $v \ll v^{*}$ ), with the results shown in Figure 4. We observe that increasing $\theta$ causes a relatively faster decline in the permeability ratio. This is to be expected, because increasing $\theta$ results in greater particle deposition, and thereby greater damage for the same number of pore volumes injected. An observation of the effluent concentration profiles, Figure 5, confirms this argument; lower values of $\theta$ have higher effluent particle concentrations. Also for $\theta=2.5$, the effluent concentration goes through a maximum. This is because of the increase in capture efficiency described earlier. The first set of particles passes through the clean bed, and a certain fraction of the particles deposits within the bed. These deposited particles act as capture sites for particles entering thereafter, thus increasing the capture efficiency. This increase in capture efficiency reduces the effluent concentration of particles. The maximum is not noticeable for relatively low or high values of the effluent concentration. For relatively low values of the effluent concentration, changes in the capture coefficient do not significantly affect the deposition rate, which is already relatively high. On the other hand, for relatively high values of the effuent concentration, the rate of deposition is fairly small and does not alter the initial capture coefficient too much.

The effect of $v / v^{*}$ on the permeability response is demonstrated in Figure 6 for a fixed value of $\theta_{0}$ and varying $v / v^{*}$. A 


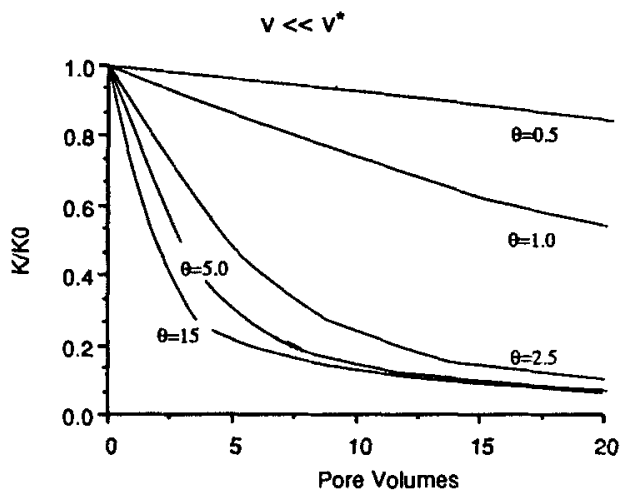

Figure 4. Effect of $\theta$ parameter on permeability response.

relatively low value of $v^{*}$ implies that conditions are unfavorable for deposition, leading to a small decline in the permeability ratio. The effect of $v / v^{*}$ on effluent concentration is studied indirectly, in terms of the filter coefficient ratio, in the following section.

\section{Filter coefficient ratio}

The filter coefficient ratio, $\lambda / \lambda_{0}$, is the ratio of the volume of particles captured in a small volume element of the network, to the volume of particles entering that element. Predicted values of this ratio near the inlet of the network are shown in Figure 7 for two different values of $v / v^{*}$. Notice that for $v / v^{*}=0.05$ the filter coefficient goes through a maximum, while for $v / v^{*}=$ 0.15 no such maximum is observed. These trends are in qualitative agreement with the experimental observations of Deb (1969), Mackie et al. (1987), and Heertjes and Lerk (1967), where experiments by some researchers showed an initial increase in the filter coefficient, while others observed only a monotonic decrease.

The filter coefficient initially increases (for $v / v^{*}=0.05$ ) due to an improvement in the particle capture efficiency because of deposited particles acting as collectors for other particles. However, as filtration proceeds, the velocity in the bonds increases (for the case of constant injection rate), and eventually the exponential term in Eq. 8 dominates. The increasing velocity makes the argument of the exponential term relatively more negative, resulting in a decrease in the filter coefficient ratio. For $v / v^{*}=$ 0.15 , the velocity term is significant from the start of the run,

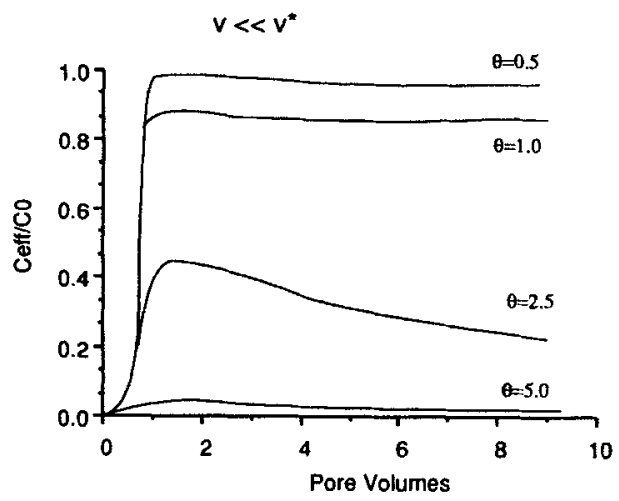

Figure 5. Effect of $\theta$ parameter on effiuent particle concentration.

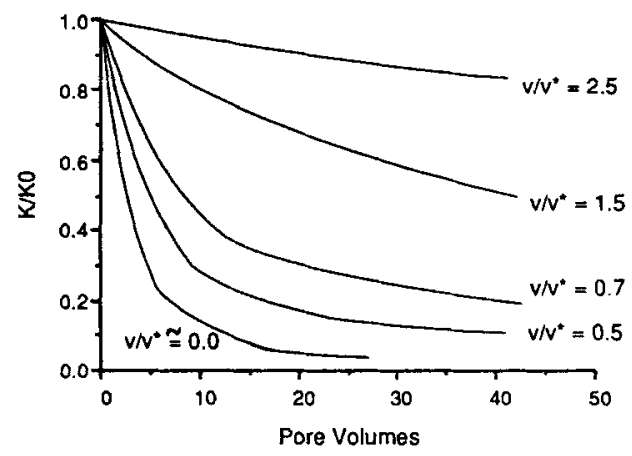

Figure 6. Effect of $v / v^{*}$ on the permeability response for $\theta_{0}=5.0$.

Values of $v / v^{*}$ shown are those at the start of a run

and therefore, an increase in the fluid velocity results in a monotonic decrease in the filter coefficient.

The reason for a maximum occurring in certain experiments and not in others has eluded researchers in the past. To explain the observed differences, previous approaches have considered several empirical relationships for the evolution of the filter coefficient (see reviews by Herzig et al. 1970; Tien and Payatakes, 1979). By changing the values of certain constants in these relationships, it is possible to observe either a maximum or a monotonic decrease in the filter coefficient ratio. In the trajectory analysis model, a maximum in the filter coefficient ratio is achieved by changing the deposition mode at some arbitrary value of deposit volume. Here the monotonically decreasing type of curves cannot be predicted. As such, existing theories do not provide an adequate understanding of the conditions governing the evolution of the filter coefficient.

The current model is unique, in that it can predict both types of curves described above without changing the deposition mode or the value of any parameter during a run. Model predictions suggest that the velocity used in an experiment is a major factor in determining the evolution of the filter coefficient. A relatively high velocity causes a monotonic decrease in $\lambda / \lambda_{0}$ with time, while intermediate values of $v / v^{*}$ cause the filter coefficient to initially increase and then decrease rapidly.

\section{Emulsion droplets vs. solid particles}

The effect of deposition morphologies was determined by simulating solid particle and emulsion droplet capture in the net-



Figure 7. Evolution of filter coefficient at inlet with time, for $\theta_{0}=5.0$.

Maximum occurs when $\nu^{*}$ is relatively large; monotonic decrease is observed when $v^{*}$ is small 


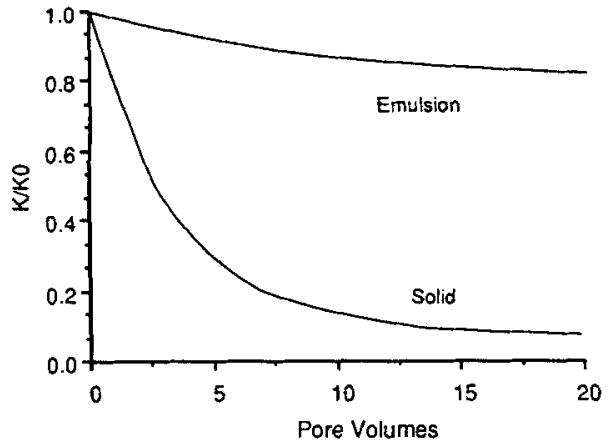

Figure 8. Effect of deposition morphologies on permeability response.

Since solid particles form dendrites, they cause a greater decline in permeability; emulsion drops form monolayer surface coverage

work. Due to the formation of dendrites, solid particles cause relatively greater decline in the formation permeability than is observed with emulsion droplets, Figure 8 . In both the runs, conditions such as the capture probability, the pore size, the particle and droplet size, and others were identical. However, the mode of calculating the pressure drop changes for solid particles was different from that used in the case of emulsion drops, as has been described earlier. The trends in Figure 8 are qualitatively similar to those observed by Soo and Radke (1985) and quantitative comparisons are made later in this paper.

\section{Effect of size distributions}

The simulations discussed so far have been performed for monodispersed pores and particles/drops. The effects of both particle and pore size size distributions on the filtration process were also examined. Studies showed that wide particle size distributions caused damage in fewer pore volumes than did relatively narrow size distributions with the same mean size. For example, changing the standard deviation from 0.0 to 2.0 (for a mean particle size of $1.0 \mu \mathrm{m}$, mean pore size of $25.0 \mu \mathrm{m}$, and a pore size standard deviation of 0.5 ) caused the pore volumes required for a $50 \%$ permeability reduction to change from 25.0 to 6.5 (Rege 1988).

\section{Comparison with experimental data}

Experimental data obtained by Soo and Radke $(1984,1985)$ and Baghdikian and Sharma (1987) were used to test the model,

Table 1. Pore Size and Emulsion Drop Size Distributions for Various Experimental Runs of Soo and Radke (1985)

\begin{tabular}{cccc}
\hline $\begin{array}{c}\text { Pore Size } \\
\text { Distribution }\end{array}$ & $\begin{array}{c}\text { Mean Drop } \\
\text { Diameter } \\
\mu_{d}, \mu \mathrm{m}\end{array}$ & $\begin{array}{c}\text { Standard } \\
\text { Deviation, } \\
\sigma_{d}\end{array}$ \\
\hline Geometric Mean & Run 1 & 3.0 & 0.23 \\
$\mu_{p o}=15.3 \mu \mathrm{m}$ & Run 2 & 5.1 & 0.35 \\
Corrected Std. Dev. & Run 3 & 9.0 & 0.56 \\
$\sigma_{p o}=1.0$ & Run 4 & 2.2 & 0.18 \\
& Run 5 & 3.0 & 0.25 \\
Geometric Mean & Run 6 & 4.2 & 0.30 \\
$\mu_{p o}=25.5 \mu \mathrm{m}$ & Run 7 & 5.7 & 0.39 \\
Corrected Std. Dev. & Run 8 & 2.2 & 0.18 \\
$\sigma_{p o}=1.25$ & (solid particles) & & \\
\hline
\end{tabular}

primarily because they report the pore size and particle/droplet size distributions, as well as other information (concentration, flow rates, etc.) that is required as input to the current model. The reported pore size distributions, which have been obtained by mercury porosimetry or centrifugal methods, need to be corrected before they are assigned to the network. It has been shown by Fatt (1956), that the true pore throat size distribution has a standard deviation twice that obtained from mercury porosimetry/centrifuge data (assuming a capillary bundle-of-tubes model for the porous media). This correction was applied to the experimentally obtained standard deviation data before it was used in the simulation. Corrections to the mean throat size are small and vary from 75 to $80 \%$ depending on the distribution. This correction was neglected, and the mean value was taken to be equal to that reported by the authors.

\section{Data of Soo and Radke}

Most of the runs performed by Soo and Radke $(1984,1985)$ were for emulsion droplets. Conditions for their various runs are shown in Table 1; both pore throat and drop diameters follow log normal distributions. The standard deviations for the particle sizes in runs $1-3$ are obtained from the reported data. For runs 4-8, the standard deviations are obtained by a linear interpolation (C. J. Radke, 1987, personal communication). The volumebased geometric means are converted to number-based means before they are used in the model.

A typical permeability response curve for run 1 is shown in Figure 9. One observes that there is good agreement between model predictions and experimental observations for $\theta_{0}=1.0$ and $v \ll v^{*}$. The reason for assuming $v \ll v^{*}$ is based on the fact that the experimental runs performed by Soo and Radke (1984) at different velocities showed no difference in the permeability response; this indicates that the velocity changes have negligible effect on the permeability ratio, for the range of velocities considered (i.e., $v / v^{*}$ is small). The value of $\theta_{0}=1$ indicates weak attraction, which is in qualitative agreement with the experimental conditions. This value of $\theta_{0}$ is then kept constant at 1.0 when simulating all other runs in Table 1. Since conditions for deposition are not altered in these runs, the value of $\theta_{0}$ is expected to be the same for all runs. Simulation results for runs 2 and 3 are shown in Figure 10. It is interesting to note that increasing the mean drop size from 5.3 to $10.0 \mu \mathrm{m}$, Figure 10, decreased the rate of initial damage. This is attributed by Soo and Radke to the ineffectiveness of plugging by larger particles (for the same total volume), characterized by their flow restriction parameter, $\beta$. The current model predicted this surprising

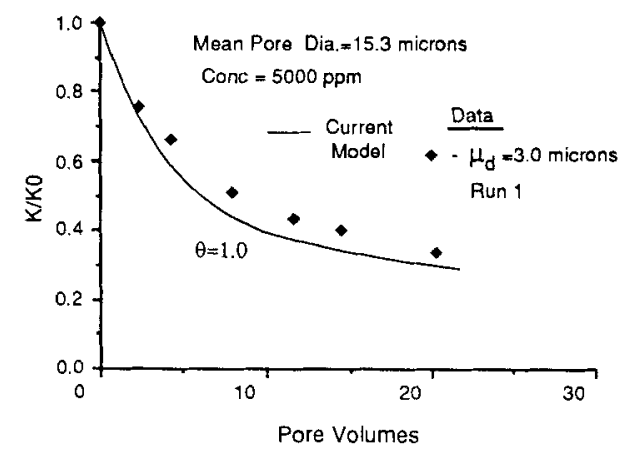

Figure 9. Permeability response curve for run 1 of Soo and Radke (1985). 


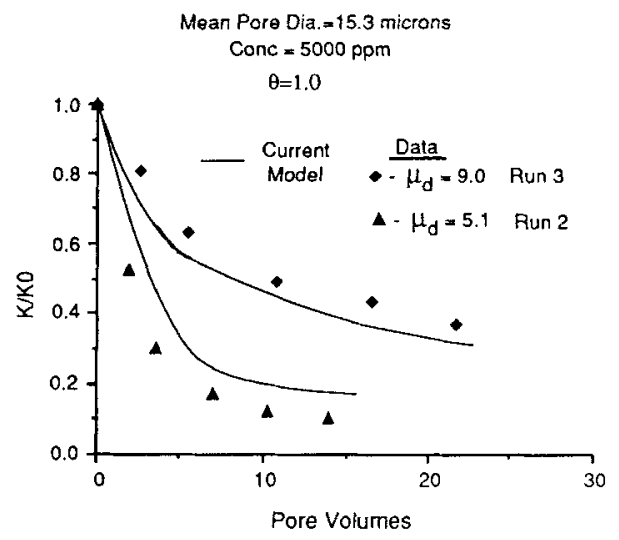

Figure 10. Permeability response date for runs 2 and 3 of Soo and Radke show suprising results.

Increasing the mean size causes a less rapid permeability decline; model predictions indicate same trends

effect without any parameter adjustment. Good agreement was also observed for runs 4,5 , and 6 with $\theta=1.0$ (Rege, 1988).

Figure 11 compares deposition of emulsion droplets to that of latex particles. Physical conditions such as surface potentials, flow rates, particle concentrations, and pore and particle/drop size distributions are identical in both the runs. The model can indeed predict the differences in the permeability response observed experimentally between solid particles and emulsion drops. The model predictions for the effluent concentration histories, Figure 12, are also in agreement with experimental data. While the solid particle concentration exhibits a maximum (for reasons stated previously), the emulsion droplet concentration in the effluent initially increases and then levels off. This leveling off occurs due to the fact that the surfaces of the bonds in the network are covered with emulsion drops, and no further deposition can occur. Entering drops pass through the network without being captured.

\section{Data of Baghdikian et al.}

Baghdikian et al. (1987) injected suspensions of kaolinite and bentonite particles into Ottawa sandpacks. The reported pore throat size distributions are fitted with a log normal distribution having a geometric mean of $18.0 \mu \mathrm{m}$ and a corrected standard

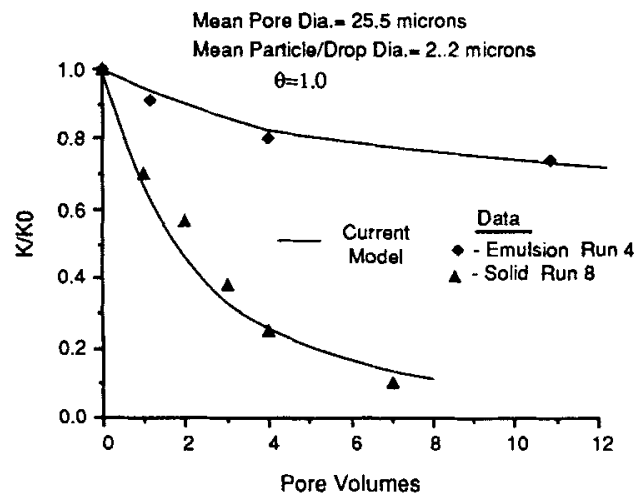

Figure 11. Solid particles exhibit different behavior than emulsion drops under identical conditions.

Solid particles form dendrites which cause a relatively rapid decline in permeability

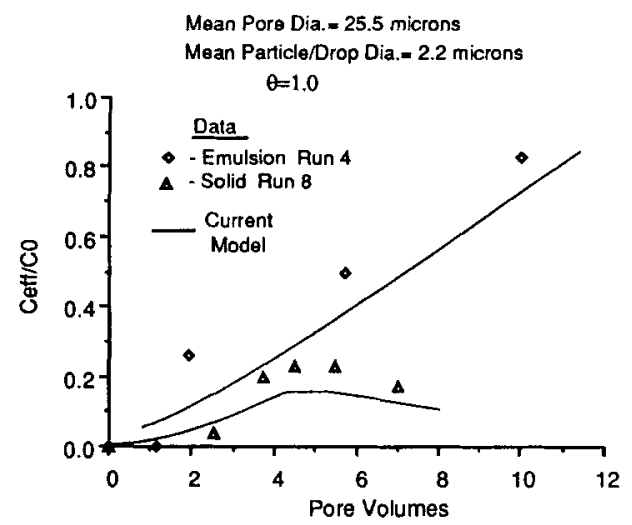

Figure 12. Dimensionless effluent concentration curves exhibit different characteristics for solids and emulsions.

In solids, dendrite formation increases capture efficiency, resulting in greater deposition

deviation of 0.85 . The particle diameters have a geometric mean of $0.02 \mu \mathrm{m}$ (in $0.01 \mathrm{M} \mathrm{KCl}$ ) and $0.2 \mu \mathrm{m}$ (in $0.1 \mathrm{M} \mathrm{KCl}$ ), while the standard deviation is about $1.5 \mu \mathrm{m}$. Other data and experimental details are well documented by Baghdikian and coworkers.

The simulations are performed using the above parameters and the results are shown in Figures 13 to 16 .

Effect of Ionic Strength. To study the effect of ionic strength, simulations were performed with different values of $\theta$. The effect of velocity changes were neglected for these runs. Increasing the ionic strength improves the conditions for deposition, resulting in a rapid decline in the permeability ratio, as shown in Figure 13. As was discussed earlier, when conditions for deposition are favorable (relatively high ionic strength), $\theta$ should have a relatively higher value than during unfavorable conditions. Such was the case in Figure 13.

Effect of Particle Concentration. Plots of the permeability ratio vs. pore volumes injected are shown in Figures 14 and 15 for different inlet particle concentrations. Notice that for the experimental data in Figure 14, decreasing the particle concentration by half reduces the rate of permeability decline also by half. Therefore, if the abcissa were changed to Pore Volumes of Particles instead of Pore Volumes, the two sets of data in Figure 14 would overlap. This suggests that the mechanism of capture

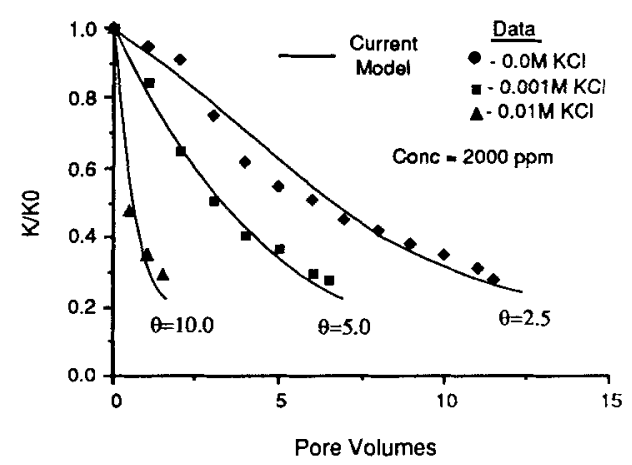

Figure 13. Model predictions and experimental data of Baghdikian et al. (1987) for bentonite suspensions at various ionic strengths. 




Figure 14. Effects of kaolinite particle concentration: simulation and data of Baghdikian et al. (1987).

Both curves are modeled with a fixed value of $\theta$

does not change when the concentration is increased from 1,000 to $2,000 \mathrm{ppm}$. For these relatively low concentrations, the same value of $\theta$ successfully predicts the permeability response.

On the other hand, in Figure 15, increasing the concentration from 2,000 to $4,000 \mathrm{ppm}$ causes a decline in the permeability that is greater than can be accounted for by changing the abcissa as above. For example, if the $2,000 \mathrm{ppm}$ suspension caused a 50\% reduction in the permeability in about six pore volumes, then 4,000 ppm should have caused the same damage in about three pore volumes. However, experiments show that only 1.25 pore volumes were required. This indicates that at higher concentrations capture mechanisms may be changing because of greater particle-particle interactions; mechanisms such as logjamming (Khilar et al., 1983) can increase the probability of capture and result in faster damage. This increase in the capture probability is accounted for in the model by an increase in the value of $\theta$.

Effect of Fluid Velocity. Experimental runs performed at different velocities provide an opportunity to test the exponential relationship used for $\theta$, that is,

$$
\theta=\theta_{0} \exp \left(-v / v^{*}\right)
$$

We first estimate $\theta_{0}$ by assuming that the experimental run performed at $0.008 \mathrm{~cm} / \mathrm{s}$, in Figure 16, is at relatively low velocities (such that the exponential term can be neglected in the above

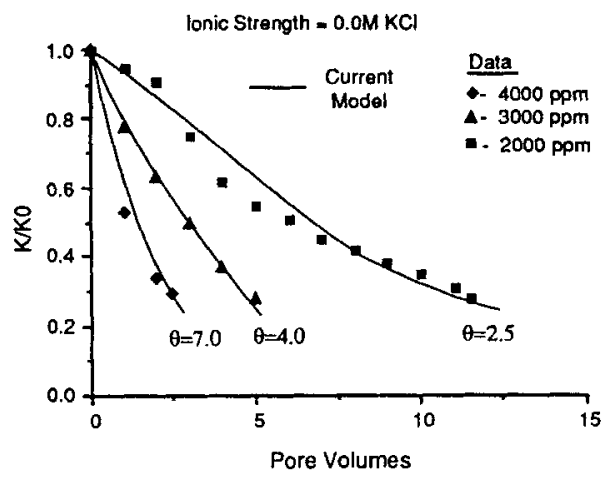

Figure 15. Concentration effects on permeability response for bentonite particles.

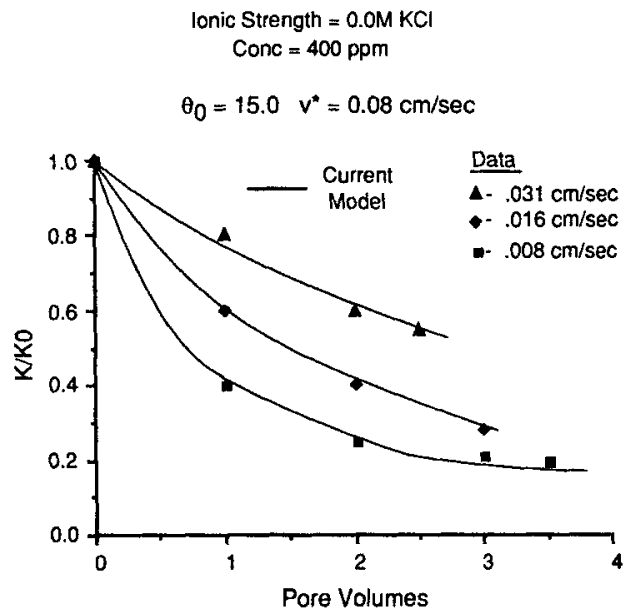

Figure 16. Effects of fluid velocity on permeability response.

Data shown are for bentonite particles from Baghdikian et al. (1987).

relationship), and fitting model predictions to experimental data. Then, keeping $\theta_{0}$ fixed, we vary $v^{*}$ such that the model predictions agree with the experimental data for the second run (at fluid velocity of $0.016 \mathrm{~cm} / \mathrm{s}$ ). The values of $\theta_{0}$ and $v^{*}$ are found to be 15.0 and $0.08 \mathrm{~cm} / \mathrm{s}$, respectively. Having estimated these values we then predict the permeability response for the run carried out at $0.031 \mathrm{~cm} / \mathrm{s}$. From Figure 16 we observe that the predicted curve agrees well with experimental data.

Comparison of model predictions with experimental data for both solids and emulsions indicates that the current model is good for simulating deep bed filtration. The parameter, $\theta_{0}$, was found to be constant (as expected) for all runs in which the conditions for deposition were not altered. $\theta_{0}$ was found to increase with increasing ionic strength of the fluid, and increasing inlet particle concentration (for relatively concentrated suspensions). Since these conditions tend to relatively increase deposition, an increase in $\theta_{0}$ is also expected.

\section{Conclusions}

The network model that has been developed is versatile in that it can predict the permeability response, effluent concentration profiles, and the evolution of the filter coefficient with time. Furthermore, it accounts for different deposition morphologies such as dendrite formation with solid particles and smooth monolayer coverage with emulsion droplets. A single parameter, $\theta$, is necessary to characterize the conditions for capture; conditions favoring greater deposition result in relatively high values of $\theta$. Comparison of model predictions with experimental data showed that if the pore size distributions and/or the particle size distributions were changed, a fixed value of $\theta$ would be adequate to model all the runs. Also, at relatively low particle concentrations, increasing the concentration has little effect on $\theta$. Other conditions that were found to affect $\theta$ are fluid velocity and ionic strength.

\section{Acknowledgment}

This research was supported by the Industrial Affiliates program at the University of Michigan, and by National Science Foundation Grant No. CBT 8516458 . Simulations were performed on the Cray X-MP supercomputer at the Pittsburgh Supercomputing Center. We acknowl- 
edge C. J. Radke at the University of California, Berkeley, for providing us with unpublished data and details of his experimental work. We also express appreciation to M. L. Hoefner and R. N. Vaidya for valuable discussions.

\section{Notation}

Units: L, length; $M$, mass; $T$, time $a=$ particle radius, $L$

$A_{1}, A_{2}, A_{3}, B_{1}=$ constants

$c, C=$ particle concentration, $M L^{-3}$

$C_{e f f}=$ effluent particle concentration, $M L^{-3}$

$D_{t}=$ transition pore throat diameter, $L$

$K$ - permeability, $L^{2}$

$P=$ pressure, $M L^{-1} \cdot T^{-2}$

$R_{0}=$ bond radius, $L$

$t=$ time

$u_{0}=$ superficial velocity, $L T^{-1}$

$U=$ centerline velocity in laminar flow, $L T^{-1}$

$v=$ interstitial velocity, $L T^{-1}$

$v^{*}=$ critical velocity, $L T^{-1}$

$x=$ axial distance, $L$

\section{Greek letters}

$\alpha=$ flow redistribution parameter

$\beta$ - flow restriction parameter

$\beta_{1}, \beta_{2}=$ parameters

$\Delta=$ difference

$\phi=$ porosity

$\lambda=$ filter coefficient, $L^{-1}$

$\mu_{t}=$ volume-based geometric mean diameter of drops, $L$

$\mu_{p a}=$ volume-based geometric mean diameter of particles, $L$

$\mu_{p o}=$ volume-based geometric mean diameter of pores, $L$

$\eta=$ viscosity, $M L^{-1} \cdot T^{-1}$

$\theta, \theta_{0}=$ parameter

$\sigma=$ particle retention, $L^{3}$ particles $/ L^{3}$ bed

$\sigma_{d}=$ standard deviation of emulsion drops

$\sigma_{p a}=$ standard deviation of solid particles

$\sigma_{p o}=$ standard deviation of pores

$\zeta=$ fraction of monolayer coverage

\section{Subscripts}

$$
\begin{aligned}
d & =\text { drop } \\
o & =\text { original condition } \\
i & =\text { interception } \\
p a & =\text { particle } \\
p o & =\text { pore } \\
s & =\text { straining } \\
x & =\text { local value at distance } x
\end{aligned}
$$

\section{Literature Cited}

Baghdikian, S. Y., M. M. Sharma, and L. L. Handy, "Flow of Clay Suspensions through Porous Media,"SPE 16257, (Feb., 1987).

Deb, A. K., "Theory of Sand Filtration," Proc. ASCE, J. Sanitary Eng. Div., 95, 399 (1969).

Dullien, F. A. L., and V. K. Batra, "Determination of the Structure of Porous Media," Ind. Eng. Chem., 62(10), 25 (1970).

Fan, L. T., R. Nassar, S. H. Hwang, and S. T. Chou, "Analysis of Deep Bed Filtration Data: Modeling as a Birth-Death Process," AIChE J., 31(11), 1781 (Nov. 1985).

Fatt, I., "The Network Model of Porous Media. I, II, III," Pet. Trans., 207, 144 (1956).

Gruesbeck, C., and R. E. Collins, "Entrainment and Deposition of Fine Particles in Porous Media," SPE J., 847 (1982).

Happel, J., and H. Brenner, Low Reynolds Number Hydrodynamics, Noordhoff, Leyden, The Netherlands, 320 (1973).

Heertjes, P. M., and F. F. Lerk, "The Function of Deep-Bed Filters," Trans. Inst. Chem. Eng., 45, T129 (1967).

Herzig, J. P., D. M. Leclerc, and P. LeGoff, "Flow of Suspensions through Porous Media-Application to Deep Bed Filtration," Ind. Eng. Chem., 62(5), 8 (1970).

Hoefner, M. L., and H. S. Fogler, "Pore Evolution and Channel Forma- tion During Flow and Reaction in Porous Media," AIChE J., 34(1), 45 (Jan. 1988)

Horner, R. M. W., R. J. Jarvis, and R. I. Mackie, "Deep Bed Filtration-A New Look at the Basic Equations," Water Res., 20, 115 (1984).

Hsu, E. H., and L. T. Fan, "Experimental Study of Deep Bed Filtration: A Stochastic Treatment," AlChE J., 30(3), 267 (March 1984).

Imdakm, A. O., and M. Sahimi, "Transport of Large Particles in Flow Through Porous Media," Phys, Rev., A36, 5304 (1987).

Ison, C. R., and K. J. Ives, "Removal Mechanisms in Deep-Bed Filtration," Chem. Eng. Sci., 24, 717, (1969).

Ives, K. J., "Rational Design of Filters," Proc. Inst. Civil Engrs. (London), 16, 189 (1960).

Ives, K. J., and V. Pienvichitr, "Kinetics of the Filtration of Dilute Suspensions," Chem. Eng. Sci., 20, 965, (1965).

Iwasaki, T., "Some Notes on Sand Filtration," J. Am. Water Works Assoc., 29, 1591 (1937).

Jerauld, G. R., J, C. Hatfield, L. E. Scriven, and H. T. Davis, "Percolation and Conduction of Voronoi and Triangular Networks: A Case Study in Topological Disorder," J. Phys. Chem., 17, 3429 (1984).

Khilar, K. C., H. S. Fogler, and J.S. Ahluwalia, "Sandstone Water Sensitivity: Existence of a Critical Rate of Salinity Decrease for Particle Capture," Chem. Eng. Sci., 38(5), 789 (1983).

Kirkpatrick, S., "Percolation and Conduction," Rev. Mod. Phys., 45(4) 574 (Oct., 1973).

Koplik, J., "On the Effective-Medium Theory of Random Linear Networks," J. Phys. Chem.: Solid State Phys., 14, 4821 (1981).

, "Creeping Flow in Two-Dimensional Networks," $J$. Fluid Mech., 119, 219 (1982).

Litwiniszyn, J., "Colmatage-Scouring Kinetics in the Light of Stochastic Birth-Death Process," Bull. Acad. Pol. Sci., Ser. Sci. Techn., 14(9), 561 (1966).

Mackie, R. I., R. W. Horner, and R. J. Jarvis, "Dynamic Modeling of Deep-Bed Filtration," AIChE J., 33(11), 1761 (Nov., 1987).

Maroudas, A., and P. Eisenklam, "Clarification of Suspensions: A Study of Particle Deposition on Granular Media. I: Some Observations on Particle Deposition," Chem. Eng. Sci., 20, 867 (1965a).

, "Clarification of Suspensions: A Study of Particle Deposition on Granular Media. II: A Theory of Clarification," Chem. Eng. Sci., 20, 875 (1965b).

Payatakes, A. C., "Model of Transient Aerosol Particle Deposition in Fibrous Media with Dendritic Pattern," AIChE J., 23(3), 192 (Mar., 1977).

Payatakes, A. C., C. Tien, and R. M. Turian, "A New Model for Granular Porous Media. I: Model Formulation," AIChE J., 19(1) 58 (Jan. 1973).

_ "Trajectory Calculation of Particle Deposition in Deep Bed Filtration," AIChE J., 20(9), 900 (Sept. 1974).

Pendse, H., and C. Tien, "A Simulation Model of Aerosol Collection in Granular Media," J. Colloid Interf. Sci., 87(1), 225 (1982).

Rege, S. D., "A Network Model for Flow, Reaction, and Particle Entrapment in Porous Media," Ph.D. Thesis, Univ. Michigan (1988).

Rege, S. D., and H. S. Fogler, "Network Model for Straining Dominated Particle Entrapment in Porous Media," Chem. Eng. Sci., 42(7), 1553 (1987).

Saffman, P. G., "The Lift on a Small Sphere in a Slow Shear Flow," $J$. Fluid Mech., 22(2), 385 (1965).

Sahimi, M., H. T. Davis, and L. E. Scriven, "Dispersion in Disordered Porous Media," Chem. Eng. Commun., 23, 329 (1983).

Sahimi, M., B. D. Hughes, L. E. Scriven and H. T. Davis, "Dispersion in Flow Through Porous Media. I, II," Chem. Eng. Sci., 41, 2103, 2123 $(1986 a, b)$

Sharma, M, M., and Y. C. Yortsos, "Transport of Particulate Suspensions in Porous Media: Model Formulation," AIChE J., 33(10), 1636 (Octo. 1987a).

"A Network Model for Deep Bed Filtration Processes," AIChE J., 33(10), 1644 (Octo. 1987b)

-, "Fines Migration in Porous Media," AIChE J., 33(10), 1654 (Octo. 1987c).

Siegel, R. A., and R. Langer, "A New Monte Carlo Approach to Diffusion in Constricted Porous Geometries," J. Colloid Interf. Sci., 109, 426 (1986).

Simon, R., and F. J. Kelsey, "The Use of Capillary Tube Networks in Reservoir Performance Studies 1," SPE J., 99 (June, 1971). 
"The Use of Capillary Tube Networks in Reservoir Performance Studies II," SPE J., 345 (Aug., 1972).

Soo, H., and C. J, Radke, "The Flow Mechanism of Dilute, Stable Emulsions in Porous Media," Ind. Eng. Chem. Fundam., 23(3), 342 (1984).

"Flow of Dilute Stable Liquid and Solid Dispersions in Underground Porous Media," AlChE J., 31(11), 1926 (Nov. 1985).

"A Filtration Model for the Flow of Dilute, Stable Emulsions in Porous Media. I: Theory," Chem. Eng. Sci, 41(2), 263 (1986).

Soo, H., M. C. Williams, and C. J. Radke, "A Filtration Model for the Flow of Dilute, Stable Emulsions in Porous Media II: Parameter Evaluation and Estimation," Chem. Eng. Sci., 41(2), 273 (1986).

Spielman, L. A., and J. A. Fitzpatrick, "Theory for Particle Collection under London and Gravity Forces," J. Colloid Interf. Sci., 42(3), 607 (1973).

Spielman, L. A., and P. M. Cukor, "Deposition of Non-Brownian Par- ticles under Colloidal Forces," J. Colloid Interf. Sci., 43(1), 51 (1973).

Stein, P. C., "A Study of the Theory of Rapid Filtration of Water through Sand," D.Sc. Diss., Mass. Inst. Tech., Cambridge (1940).

Tien, C., and A. C. Payatakes, "Advances in Deep Bed Filtration," AIChE J., 25(9), 737 (Sept., 1979).

Tien C., R. M. Turian, and H. Pendse, "Simulation of the Dynamic Behavior of Deep Bed Filters," AIChE J., 25(3), 385 (Mar., 1979).

Todd, A. C., J. E. Somerville, and G. Scott, "The Application of Depth of Formation Damage Measurements in Predicting Water Injectivity Decline," SPE 12498 (Feb., 1984).

Yortsos, Y. C., and M. M. Sharma, "Application of Percolation Theory to Noncatalytic Gas-Solid Reactions," AIChE J., 32(1) 46 (Jan. 1986).

Manuscript received Apr. 7, 1988, and revision received June 29, 1988. 